\title{
Association between periodontal disease and non-apnea sleep disorder: a systematic review
}

\author{
Schmidlin, Patrick R ; Khademi, Abbasali ; Fakheran, Omid
}

\begin{abstract}
Objectives: Inflammation is an integral part of the pathogenesis of periodontitis and sleep disorders. The aim of the present study was to review systematically the current evidence relating to the association between periodontal diseases and non-apnea sleep disorder. Materials and methods: Systematic searches were performed in MEDLINE, PsycINFO, Cochrane library, Web of Science, and Scopus without any limitation. Following preliminary screening, the quality of the remaining selected papers was appraised using the Newcastle-Ottawa Scale. Due to substantial heterogeneity among the selected articles, main outcomes were reported in a qualitative manner. Results: Following screening and evaluation, a final set of 13 studies was selected for inclusion. These studies examined the association between periodontal disease and short sleep duration, long sleep duration, poor sleep quality, or non-specific sleep disorders. The majority $(\mathrm{N}=12 / 13)$ reported an association or trend between one type of sleep abnormality and periodontal or gingival parameters. Conclusion: Despite the respective limitations of the articles included in this systematic review, an association between periodontal diseases and sleep disturbances was apparent. Clinical relevance: Adequate management of periodontal disease requires that a patient's lifestyle factors be taken into consideration in treatment planning. One such factor is sleep initiation and maintenance. An obvious association between sleep disturbances and periodontitis exists. Sleep disorders may induce systemic inflammation, which, in turn, could influence the development of periodontitis. Keywords: Periodontal disease; Sleep disorders; Systematic review.
\end{abstract}

DOI: https://doi.org/10.1007/s00784-020-03475-2

Posted at the Zurich Open Repository and Archive, University of Zurich

ZORA URL: https://doi.org/10.5167/uzh-194454

Journal Article

Accepted Version

Originally published at:

Schmidlin, Patrick R; Khademi, Abbasali; Fakheran, Omid (2020). Association between periodontal disease and non-apnea sleep disorder: a systematic review. Clinical Oral Investigations, 24(10):3335-3345.

DOI: https://doi.org/10.1007/s00784-020-03475-2 
Title:

Association of periodontal disease and Non-Apnea Sleep Disorder: A systematic review

Short running title:

Association of Periodontitis and sleep

Full names of the authors:

Omid Fakheran ${ }^{1}$, Abbasali Khademi ${ }^{2}$, Patrick R. Schmidlin ${ }^{3}$

Author's institutional affiliations:

1. Dental research center, Department of Periodontics, Dental research institute, Isfahan University of Medical sciences, Isfahan, Iran

2. Dental research center, Department of Endodontics, Dental research institute, Isfahan University of Medical sciences, Isfahan, Iran

3. Clinic of Conservative and Preventive Dentistry, Center of Dental Medicine, University of Zurich, Plattenstrasse 11 8032, Zurich, Switzerland.

Conflict of Interest Statement

The authors declare no conflict of interest.

Corresponding Author:

Patrick R. Schmidlin

Clinic of Conservative and Preventive Dentistry, Center of Dental Medicine, University of Zurich, Plattenstrasse 11 8032, Zurich, Switzerland

Email: patrick.schmidlin@zzm.uzh.ch 
Abstract and keywords

Aim: Inflammation is an integral part of pathogenesis in both periodontitis and sleep disorders. The aim of this study is to systematically review the current evidence regarding the association between periodontal diseases and non-apnea sleep disorder.

Materials and Methods: Systematic search were performed in MEDLINE, PsycINFO, Cochrane library, Web of Science and, Scopus without any limitation. Following screening the papers, the quality of papers were assessed by the Newcastle-Ottawa Scale. Based on substantial heterogeneity among the selected articles, we reported the main outcomes in a qualitative manner.

Results: Following screening and evaluating the papers, a final set of 13 studies were included in this systematic review. These papers evaluated the association of periodontal disease with short sleep duration; long sleep duration, low sleep quality or non-specific sleep disorders. Almost all of the studies $(\mathrm{N}=12 / 13)$ reported an association or trend regarding one type of sleep abnormality with periodontal or gingival parameters.

Conclusion: Despite the respective limitations of the articles included within this review, there appears to be an association between periodontal diseases and sleep disturbances.

Key words: Systematic review; periodontal disease; sleep disorders

\section{Clinical Relevance:}

Scientific rationale for the study: The association of sleep disturbances and periodontal disease is insufficiently clear to provide sharp recommendations in clinical practice. This systematic review provides an evidence summary of this association.

Principal findings: It is obvious that there is an association between sleep disturbances and periodontal diseases. Sleep disorders may potentially induce systemic inflammation and this could have an effect in the development of periodontitis.

Practical implications: Clinicians should consider the main life style factors of their patients in the way of managing the periodontal disease. One of the emerging factors in this regard is sleep Initiation and maintenance disorders. 


\section{Main Text}

\section{Introduction:}

During the last decade, evidence has been growing regarding the association of health and sleep. It has been documented that sleep Initiation and maintenance disorders, such as extremes of sleep duration and insomnia adversely influence risk of inflammatory disease and contribute to all-cause mortality (Dew et al., 2003; Kripke, Garfinkel, Wingard, Klauber, \& Marler, 2002; Mallon, Broman, \& Hetta, 2002; Vgontzas, Fernandez-Mendoza, Liao, \& Bixler, 2013). Scientists suggested a U-shaped association of sleep duration and overall mortality (Cai et al., 2015; W. J. Lee, Peng, Liang, Chiou, \& Chen, 2017). Many epidemiological studies showed that sleep duration of about seven hours was associated with lowest mortality rate (Francesco $P$ Cappuccio, Lanfranco D'Elia, Pasquale Strazzullo, \& Michelle A Miller, 2010; Panel et al., 2015).

Sleep deprivation has been independently linked to several diseases, particularly to diabetes mellitus, metabolic syndrome, hypertension and coronary artery disease (Kim et al., 2015; King et al., 2008; Yaggi, Araujo, \& McKinlay, 2006). Increasing the level of systemic inflammatory and oxidative stress biomarkers have been introduced as the causal pathway of this association between the mentioned pathologic conditions and short sleep duration (Theresanne DeMartino et al., 2016).Recently, also the association between short sleep duration with the risk of systemic infections has been highlighted, explained by the experimental evidence of host immunity(Luciana Besedovsky et al., 2019; Bollinger et al., 2009).

It is interesting that the long duration of sleep also have some impact on cardiovascular outcomes(Cappuccio, Cooper, D'Elia, Strazzullo, \& Miller, 2011). the relationship between quantity of sleep and incidence of type 2 diabetes mellitus was assessed in a study, and it was shown that higher relative risk of type 2 diabetes mellitus was noted both for short duration of sleep ( $\leq 5-6$ hours/night) and long duration of sleep (>8-9 hours/night) (F. P. Cappuccio, L. D'Elia, P. Strazzullo, \& M. A. Miller, 2010a). In a systematic review the authors reported that both long and short durations of sleep are significant predictors of death in prospective population studies (F. P. Cappuccio, L. D'Elia, P. Strazzullo, \& M. A. Miller, 2010b). Sleep disturbances, including insomnia, independently contribute to the risk of inflammatory disorders and major depressive disorder (Irwin \& Opp, 2017).

The mechanisms by which non-apnea sleep disorder (NA-SD) (excluding apnea sleep disorder or named obstructive sleep apnea disorder) influence systemic health are unclear, but some articles have suggested that altered sleep might affect the level of various inflammatory markers such as tumor necrosis factor (TNF), C-reactive protein (CRP) and interleukins which regulates inflammatory responses(Haack, Sanchez, \& Mullington, 2007; Meier-Ewert et al., 2004; Sanjay R Patel et al., 2009).

On the other side, periodontal disease refers to the destructive inflammatory reaction of the surrounding tissues of the tooth such as the alveolar bone, cementum, periodontal ligaments and gingival tissues. In periodontitis, microbial factors induce a series of host responses that 
mediate inflammatory events. In susceptible individuals, dysregulation of inflammatory and immune pathways leads to chronic inflammation, tissue destruction, tooth loosening, and tooth loss (Cekici, Kantarci, Hasturk, \& Van Dyke, 2014). These diseases are prevalent and can affect up to $90 \%$ of the worldwide population(Pihlstrom, Michalowicz, \& Johnson, 2005).

Since inflammation is characteristic of both periodontitis and sleep disorders, sleep may have a role in periodontal disease. To the best of our knowledge, no systematic review has been published based on this question in scientific literature. The aim of this review is to systematically assess the association between periodontal diseases and (NA-SD) to clarify this issue and creating a foundation for future studies.

\section{MATERIALS AND METHODS}

\section{Protocol development and focused question:}

This systematic review was conducted according to the Cochrane Handbook (Higgins \& Green, 2011)and reported according to the PRISMA statement recommendations(Moher, Liberati, Tetzlaff, \& Altman, 2009). A protocol was developed and submitted to PROSPERO database (ID: 169878) (www.crd.york.ac.uk/PROSPERO).

The literature search was conducted to answer the following focused question: "Is there any association between periodontal disease and Non-Apnea Sleep Disorder in adult individuals?"

Following the PICO criteria, all adult subjects (Age $\geq 19$ years) were defined as the population. Any type of periodontal disease was considered as intervention/exposure. Individuals without any periodontal disease defined as comparison. Non-Apnea Sleep Disorders considered as outcome.

\section{Eligibility criteria:}

Inclusion criteria and exclusion criteria were defined a priori. The inclusion criteria were as follows: (a) English language; (b) No limitation for design of studies; (c) Diagnosing of periodontal disease based on clinical examination (not self-reported measures); (d) studies reporting any type of sleep disorder outcomes.

Exclusion criteria included (a) studies enrolling children or adolescents (Age<18 years); (b) studies on obstructive sleep apnea (OSA) because it has been systematically evaluated and reported previously(Al-Jewair, Al-Jasser, \& Almas, 2015); (c) Non-scientific commentaries, reports, letters and news articles.

\section{Information sources and search}

Electronic literature searches were performed on the following databases, encompassing much of the available published literature: MEDLINE, PsycINFO, Cochrane library, Web of Science and, 
Scopus. These sources were systematically searched up to 11 February 2020 without any restrictions on language or date of publication.

The following search terms and search protocol have been used for this systematic search: ( ( ( ( ( ( ( ( Periodontitis) OR gingivitis) OR periodontal disease) OR gum disease) OR periodontal attachment loss) OR alveolar bone loss) OR clinical attachment loss) OR clinical attachment level) OR periodontal pocket depth) OR tooth loss) OR teeth loss. The terms and key words adapted for each database as necessary. Detailed search protocol for each databases are available as supplementary material.

Following the completion of search, the references in the papers that were selected were also reviewed to include additional articles that were not found in the original electronic search. A number of websites that list ongoing clinical trials were also searched (http://clinicaltrials.gov, http://www.centerwatch.com/, and http://www.clinicalconnection.com).

\section{Data collection}

Two authors (OF and AK) independently searched through the retrieved article lists. The two sets of papers were then compared. Disagreements were resolved by discussion or, if necessary, by including a third researcher (PS) to make the final decision. Duplicate articles were excluded. One investigator (OF) extracted the data, and a second investigator (PS) checked the retrieved data independently for completeness and accuracy.

Data were extracted and assimilated on a piloted, standardized data collection sheet. Data was extracted relating to study design, country of publication, participant demographics, sample size, age range of participants, diagnostic criteria for periodontal disease, definition and measures of sleep disorders and outcomes as related to aims of this study.

\section{Risk of bias assessment}

The Newcastle-Ottawa Scale (NOS) used to assess the included studies and evaluate their quality in terms of study group selection, group comparability, and exposure or outcome of interest(Peterson, Welch, Losos, \& Tugwell, 2011). The NOS consists of nine items. Each item is assigned a star if the study meets the criteria of the item. The studies then divided into three categories: low risk of bias ( $\geq 7$ stars), moderate risk of bias (5-6 stars), high risk of bias ( $\leq 4$ stars).

\section{Data synthesis}

Data were pooled into evidence table by clinical parameters and measures of sleep disorder. A descriptive summary was created to report on study characteristics, results, and dissimilarities. Meta-analysis was not conducted because of the substantial heterogeneity in selected studies. 


\section{Results}

\section{Literature search}

A total of 700 items were identified from the selected databases, and no items were found from other sources. By screening titles and abstracts and removing duplicates, 29 papers were retrieved, for which full text versions were obtained for detailed assessment. Further examination of the reference lists in the 29 retrieved articles didn't add any paper. We excluded 16 papers based on various reasons after comprehensive reading of the full texts (Appendix Table A). Ultimately, a final set of 13 studies were included in this systematic review. Detailed information regarding the studies is summarized in table 1.

\section{General Characteristics of the Studies}

Most of the included studies have cross-sectional design $(\mathrm{N}=9)$, followed by case-control $(\mathrm{N}=3)$ and retrospective cohort study $(\mathrm{N}=1)$. We classified the results of included papers based on the sleep health parameters which have been investigated by the studies. These disorders are short sleep duration; long sleep duration, low sleep quality and non-specific sleep disorders. It should be noted that sleep health data which has been reported in all included studies are based on self-reported items by targeted individuals.

\section{Short sleep duration:}

Most of the included studies in this review $(\mathrm{N}=8)$ investigated the association of short sleep duration and periodontal disease. The results of 4 studies showed that periodontal disease was directly related to short sleep duration (Alqaderi, Goodson, \& Agaku, 2019; Carra et al., 2017; Karaaslan \& Dikilitaş, 2019; Singla et al., 2016). However, the other 4 studies reported that there is no significant association between periodontitis and sleep duration in fully-adjusted models (Beydoun et al., 2019; Saletu et al., 2005; Wiener, 2016; Yoshida, Ogawa, Imaki, Nakamura, \& Tanada, 1997).

\section{Long sleep duration:}

Two cross-sectional studies assessed the association of long sleep duration with periodontal disease(Han, Park, \& Park, 2018; Romandini et al., 2017). Both of the studies have been conducted based on the Korean National Health and Nutrition Examination Survey (KNHANES), but with different sample sizes and including criteria. The first study after adjusting results for confounding factors concluded that long sleep duration can be considered as an independent risk indicator for periodontal disease among Korean(Han et al., 2018). The other study also reported an independent direct association between sleep duration and periodontitis prevalence in a fully adjusted model(Romandini et al., 2017). This study mentioned that age and 
consumption frequency of coffee, tea, chocolate and red wine seemed to be the main confounders in this statistical association.

\section{Low sleep quality:}

Four included studies evaluated the association of periodontal disease with quality of sleep based on The Pittsburgh Sleep Quality Index (PSQI). Three of four studies have found a significant statistical association between periodontitis and quality of sleep (Grover, Malhotra, \& Kaur, 2015; Karaaslan \& Dikilitaş, 2019; Singh et al., 2019). The fourth paper also reported a trend towards an increase in sleep disorders (evaluated by the PSQI) in periodontitis patients. However this association was not statistically significant(Saletu et al., 2005).

\section{Non-specific sleep disorders:}

Among the included studies, two of them consider all type of sleep disorders as one cumulative variable and evaluated its relation with periodontal parameters(Carra et al., 2017; C. F. Lee et al., 2014). Both of these studies have been conducted among a large scale of people based on Taiwan and France national health surveys. One of this studies reported that individuals who suffering from sleep disorder have an increased risk of periodontal diseases (C. F. Lee et al., 2014). The second investigation has found that individuals with sleep disorder displayed significantly higher amount of dental plaque and higher level of gingival inflammation(Carra et al., 2017).

\section{Discussion}

Sleep is an indispensable natural phenomenon and has a substantial role in well-being by activation of systemic defense mechanisms(AlDabal \& BaHammam, 2011). Inflammatory homeostatic procedures and immunological memory development dependent on a well sleep health for normal function (Haspel et al., 2020; Ibarra-Coronado et al., 2015). Low sleep quality and duration which adversely affect human physical health and mortality risk have become increasingly common due to a 24/7 lifestyle and longer working hours (Bannai \& Tamakoshi, 2014; Coveney, 2014; Gradisar, Gardner, \& Dohnt, 2011). In This regard, sleep disorders have emerged as a critical social and medical concern (Faria, Varela, \& Gardner, 2019).

This study systematically evaluated the data published concerning the association between periodontal disease and sleep Initiation and maintenance disorders. There was found to be high levels of variability among the results in the included studies. This might be related to various sleep measures and different periodontal parameters which have been used in these studies. However it is interesting to note that almost all of the studies ( $N=12 / 13)$ reported an association or trend regarding one type of sleep abnormality with periodontal or gingival parameters. There was just one study which didn't find any association between self-reported average of daily 
sleep duration and CPITN. This study has been published 43 years ago and was conducted among a group of factory workers in Japan(Yoshida et al., 1997).

There is substantial evidence supporting the biological plausibility of a positive association between the prevalence of periodontitis and sleep disturbances. First, sleep disorders could potentially induce systemic inflammation and this could have an effect in the development of periodontitis (S. R. Patel et al., 2009; Pink et al., 2015). Second, a body with impaired immune system due to sleep Initiation and maintenance disorders could be more susceptible to infectious disease which one of them is periodontitis(Cohen, Doyle, Alper, Janicki-Deverts, \& Turner, 2009; Irwin, 2015; Slots, 2017). The third possible mechanism is a reverse causation. This could be explained by systemic inflammation which has been induced by periodontitis (Hajishengallis, 2015; Suh et al., 2019). The elevated levels of inflammatory biomarkers such as cytokines could adversely influence on the sleep health (L. Besedovsky \& Lange, 2019; Irwin, 2019).

In the way of exploring the association between sleep disorders and periodontitis, researchers should pay enough attention to confounders. Based on reviewing of the literature, there are many factors such as age, smoking, life style and systemic disease which have independently associated with both of sleep and periodontal disease (Genco \& Borgnakke, 2013; Smagula, Stone, Fabio, \& Cauley, 2016). These confounders could potentially have influenced the reported or non-reported associations in included studies. For instance, there are some evidence regarding the ethno-racial differences in association between systemic inflammation and sleep duration. $C$ reactive protein (CRP), a substantial inflammatory biomarker, has reported to be increased only in long sleepers among Asians, contrary to other racial groups which have exhibited elevated levels of CRP even in short sleepers(Dowd, Goldman, \& Weinstein, 2011; Grandner et al., 2013).

Regarding to methodological assessment, all the included studies used subjective criteria instead of objective instruments (e.g. Polysomnography or actigraphy) to assess sleep health status. However, it should be noted that objective sleep instruments are more accurate for evaluation of acute sleep curtailment. In the way of detecting the association between periodontal disease and sleep disorders, detecting chronic (long-term) sleep habits is more significant. Hence, self-reported sleep measures are more suitable for these types of studies (Bliwise \& Young, 2007; T. DeMartino et al., 2016).

A major strength of the present systematic review is that it covers all type of (NA-SD) such as sleep deprivation, long sleep duration and low quality sleep. To the best of our knowledge, this is the first systematic review so far, which has focused on the association of periodontal disease and (NA-SD).Therefore, it can be considered as a milestone for future investigations.

Nonetheless, a number of potential limitations of the review must be acknowledged. First, most of the selected studies in this review were based on cross-sectional designed investigation, which may limit the argumentation intensity of our conclusion. Second, the studies included in 
this review varied substantially in population sampled; with some recruiting from the general population and others selectively recruiting by periodontal parameters, sex, ethnicity or some other characteristic (e.g., factory workers).this introduced considerable heterogeneity into the review, thus making meta-analysis inapplicable. Accordingly, more prospective studies such as controlled randomized clinical trials need to be conducted to clarify the relationship between periodontal diseases and sleep disorders.

\section{Conclusion:}

Despite the relative limitations of the studies included within this review, there appears to be an association between sleep Initiation and maintenance disorders and periodontal diseases. Future longitudinal studies are needed for better understanding of the interplay between periodontal tissue inflammations and sleep disorders. This may help in controlling the lifestyle factors which may involve in periodontal disease pathogenesis.

\section{References}

Al-Jewair, T. S., Al-Jasser, R., \& Almas, K. (2015). Periodontitis and obstructive sleep apnea's bidirectional relationship: a systematic review and meta-analysis. Sleep and Breathing, 19(4), 1111-1120.

AlDabal, L., \& BaHammam, A. S. (2011). Metabolic, endocrine, and immune consequences of sleep deprivation. The open respiratory medicine journal, 5, 31.

Alqaderi, H., Goodson, J. M., \& Agaku, I. (2019). Association between sleep and severe periodontitis in a nationally representative adult US population. Journal of periodontology.

Bannai, A., \& Tamakoshi, A. (2014). The association between long working hours and health: a systematic review of epidemiological evidence. Scand J Work Environ Health, 40(1), 518. doi:10.5271/sjweh.3388

Besedovsky, L., \& Lange, T. (2019). The Sleep-Immune Crosstalk in Health and Disease. 99(3), 1325-1380. doi:10.1152/physrev.00010.2018

Besedovsky, L., Lange, X. T., Haack, M., Besedovsky, L., Lange, T., \& Crosstalk, S.-i. (2019). DISEASE THE SLEEP RESPONSE TO IMMUNE. 
Beydoun, H. A., Hossain, S., Beydoun, M. A., Weiss, J., Zonderman, A. B., \& Eid, S. M. (2019). Periodontal disease, sleep duration, and white blood cell markers in the 2009 to 2014 National Health and Nutrition Examination Surveys. Journal of periodontology.

Bliwise, D. L., \& Young, T. B. (2007). The parable of parabola: what the U-shaped curve can and cannot tell us about sleep. Sleep, 30(12), 1614-1615. doi:10.1093/sleep/30.12.1614

Bollinger, T., Bollinger, A., Skrum, L., Dimitrov, S., Lange, T., \& Solbach, W. (2009). Sleepdependent activity of T cells and regulatory T cells. Clinical \& Experimental Immunology, 155(2), 231-238.

Cai, H., Shu, X.-O., Xiang, Y.-B., Yang, G., Li, H., Ji, B.-T., . . Zheng, W. (2015). Sleep duration and mortality: a prospective study of 113,138 middle-aged and elderly Chinese men and women. Sleep, 38(4), 529-536.

Cappuccio, F. P., Cooper, D., D'Elia, L., Strazzullo, P., \& Miller, M. A. (2011). Sleep duration predicts cardiovascular outcomes: a systematic review and meta-analysis of prospective studies. Eur Heart J, 32(12), 1484-1492. doi:10.1093/eurheartj/ehr007

Cappuccio, F. P., D'Elia, L., Strazzullo, P., \& Miller, M. A. (2010a). Quantity and quality of sleep and incidence of type 2 diabetes: a systematic review and meta-analysis. Diabetes Care, 33(2), 414-420. doi:10.2337/dc09-1124

Cappuccio, F. P., D'Elia, L., Strazzullo, P., \& Miller, M. A. (2010). Sleep duration and all-cause mortality: a systematic review and meta-analysis of prospective studies. Sleep, 33(5), 585-592.

Cappuccio, F. P., D'Elia, L., Strazzullo, P., \& Miller, M. A. (2010b). Sleep duration and all-cause mortality: a systematic review and meta-analysis of prospective studies. Sleep, 33(5), 585-592. doi:10.1093/sleep/33.5.585

Carra, M. C., Schmitt, A., Thomas, F., Danchin, N., Pannier, B., \& Bouchard, P. (2017). Sleep disorders and oral health: a cross-sectional study. Clinical oral investigations, 21(4), 975983.

Cekici, A., Kantarci, A., Hasturk, H., \& Van Dyke, T. E. (2014). Inflammatory and immune pathways in the pathogenesis of periodontal disease. Periodontology 2000, 64(1), 57-80.

Cohen, S., Doyle, W. J., Alper, C. M., Janicki-Deverts, D., \& Turner, R. B. (2009). Sleep habits and susceptibility to the common cold. Arch Intern Med, 169(1), 62-67. doi:10.1001/archinternmed.2008.505

Coveney, C. M. (2014). Managing sleep and wakefulness in a 24-hour world. Sociol Health IIIn, 36(1), 123-136. doi:10.1111/1467-9566.12046

DeMartino, T., Ghoul, R. E., Wang, L., Bena, J., Hazen, S. L., Tracy, R., . . Mehra, R. (2016). Oxidative Stress and Inflammation Differentially Elevated in Objective Versus Habitual Subjective Reduced Sleep Duration in Obstructive Sleep Apnea. Sleep, 39(7), 1361-1369. doi:10.5665/sleep.5964

DeMartino, T., Ghoul, R. E., Wang, L., Bena, J., Hazen, S. L., Tracy, R., ... Mehra, R. (2016). Oxidative stress and inflammation differentially elevated in objective versus habitual subjective reduced sleep duration in obstructive sleep apnea. Sleep, 39(7), 1361-1369.

Dew, M. A., Hoch, C. C., Buysse, D. J., Monk, T. H., Begley, A. E., Houck, P. R., . . Reynolds III, C. F. (2003). Healthy older adults' sleep predicts all-cause mortality at 4 to 19 years of follow-up. Psychosomatic medicine, 65(1), 63-73.

Dowd, J. B., Goldman, N., \& Weinstein, M. (2011). Sleep duration, sleep quality, and biomarkers of inflammation in a Taiwanese population. Annals of epidemiology, 21(11), 799-806.

Faria, G. S., Varela, S. A. M., \& Gardner, A. (2019). The social evolution of sleep: sex differences, intragenomic conflicts and clinical pathologies. Proc Biol Sci, 286(1894), 20182188. doi:10.1098/rspb.2018.2188 
Genco, R. J., \& Borgnakke, W. S. (2013). Risk factors for periodontal disease. Periodontol 2000, 62(1), 59-94. doi:10.1111/j.1600-0757.2012.00457.x

Gradisar, M., Gardner, G., \& Dohnt, H. (2011). Recent worldwide sleep patterns and problems during adolescence: a review and meta-analysis of age, region, and sleep. Sleep Med, 12(2), 110-118. doi:10.1016/j.sleep.2010.11.008

Grandner, M. A., Buxton, O. M., Jackson, N., Sands-Lincoln, M., Pandey, A., \& Jean-Louis, G. (2013). Extreme sleep durations and increased C-reactive protein: effects of sex and ethnoracial group. Sleep, 36(5), 769-779.

Grover, V., Malhotra, R., \& Kaur, H. (2015). Exploring association between sleep deprivation and chronic periodontitis: A pilot study. Journal of Indian Society of Periodontology, 19(3), 304.

Haack, M., Sanchez, E., \& Mullington, J. M. (2007). Elevated inflammatory markers in response to prolonged sleep restriction are associated with increased pain experience in healthy volunteers. Sleep, 30(9), 1145-1152.

Hajishengallis, G. (2015). Periodontitis: from microbial immune subversion to systemic inflammation. Nat Rev Immunol, 15(1), 30-44. doi:10.1038/nri3785

Han, K., Park, Y.-M., \& Park, J.-B. (2018). Evaluation of an association between long sleep duration and periodontal disease among men and women using nationally representative data. Gaceta sanitaria, 32, 143-150.

Haspel, J. A., Anafi, R., Brown, M. K., Cermakian, N., Depner, C., Desplats, P., . . Solt, L. A. (2020). Perfect timing: circadian rhythms, sleep, and immunity - an NIH workshop summary. JCl Insight, 5(1). doi:10.1172/jci.insight.131487

Higgins, J., \& Green, S. (2011). Analysing data and undertaking metaanalyses. Cochrane Handbook for Systematic Reviews of Interventions 5.1. 0. The Cochrane Collaboration. In.

Ibarra-Coronado, E. G., Pantaleon-Martinez, A. M., Velazquez-Moctezuma, J., Prospero-Garcia, O., Mendez-Diaz, M., Perez-Tapia, M., . . . Morales-Montor, J. (2015). The Bidirectional Relationship between Sleep and Immunity against Infections. J Immunol Res, 2015, 678164. doi:10.1155/2015/678164

Irwin, M. R. (2015). Why sleep is important for health: a psychoneuroimmunology perspective. Annu Rev Psychol, 66, 143-172. doi:10.1146/annurev-psych-010213-115205

Irwin, M. R. (2019). Sleep and inflammation: partners in sickness and in health. Nat Rev Immunol, 19(11), 702-715. doi:10.1038/s41577-019-0190-z

Irwin, M. R., \& Opp, M. R. (2017). Sleep Health: Reciprocal Regulation of Sleep and Innate Immunity. Neuropsychopharmacology, 42(1), 129-155. doi:10.1038/npp.2016.148

Karaaslan, F., \& Dikilitaş, A. (2019). The association between stage-grade of periodontitis and sleep quality and oral health-related quality of life. Journal of periodontology, 90(10), 1133-1141.

Kim, J.-Y., Yadav, D., Ahn, S. V., Koh, S.-B., Park, J. T., Yoon, J., . . Lee, S.-H. (2015). A prospective study of total sleep duration and incident metabolic syndrome: the ARIRANG study. Sleep medicine, 16(12), 1511-1515.

King, C. R., Knutson, K. L., Rathouz, P. J., Sidney, S., Liu, K., \& Lauderdale, D. S. (2008). Short sleep duration and incident coronary artery calcification. Jama, 300(24), 2859-2866.

Kripke, D. F., Garfinkel, L., Wingard, D. L., Klauber, M. R., \& Marler, M. R. (2002). Mortality associated with sleep duration and insomnia. Archives of general psychiatry, 59(2), 131136. 
Lee, C. F., Lin, M. C., Lin, C. L., Yen, C. M., Lin, K. Y., Chang, Y. J., \& Kao, C. H. (2014). Non-apnea sleep disorder increases the risk of periodontal disease: a retrospective populationbased cohort study. Journal of periodontology, 85(4), e65-e71.

Lee, W. J., Peng, L. N., Liang, C. K., Chiou, S. T., \& Chen, L. K. (2017). Long sleep duration, independent of frailty and chronic inflammation, was associated with higher mortality: a national population-based study. Geriatrics \& gerontology international, 17(10), 14811487.

Mallon, L., Broman, J. E., \& Hetta, J. (2002). Sleep complaints predict coronary artery disease mortality in males: a 12-year follow-up study of a middle-aged Swedish population. $J$ Intern Med, 251(3), 207-216.

Meier-Ewert, H. K., Ridker, P. M., Rifai, N., Regan, M. M., Price, N. J., Dinges, D. F., \& Mullington, J. M. (2004). Effect of sleep loss on C-reactive protein, an inflammatory marker of cardiovascular risk. Journal of the American College of Cardiology, 43(4), 678-683.

Moher, D., Liberati, A., Tetzlaff, J., \& Altman, D. G. (2009). Preferred reporting items for systematic reviews and meta-analyses: the PRISMA statement. Annals of internal medicine, 151(4), 264-269.

Panel, C. C., Watson, N. F., Badr, M. S., Belenky, G., Bliwise, D. L., Buxton, O. M., . . G Grandner, M. A. (2015). Joint consensus statement of the American Academy of Sleep Medicine and Sleep Research Society on the recommended amount of sleep for a healthy adult: methodology and discussion. Sleep, 38(8), 1161-1183.

Patel, S. R., Zhu, X., Storfer-Isser, A., Mehra, R., Jenny, N. S., Tracy, R., \& Redline, S. (2009). Sleep duration and biomarkers of inflammation. Sleep, 32(2), 200-204.

Patel, S. R., Zhu, X., Storfer-Isser, A., Mehra, R., Jenny, N. S., Tracy, R., \& Redline, S. (2009). Sleep duration and biomarkers of inflammation. Sleep, 32(2), 200-204. doi:10.1093/sleep/32.2.200

Peterson, J., Welch, V., Losos, M., \& Tugwell, P. (2011). The Newcastle-Ottawa scale (NOS) for assessing the quality of nonrandomised studies in meta-analyses. Ottawa: Ottawa Hospital Research Institute.

Pihlstrom, B. L., Michalowicz, B. S., \& Johnson, N. W. (2005). Periodontal diseases. The Lancet, 366(9499), 1809-1820.

Pink, C., Kocher, T., Meisel, P., Dorr, M., Markus, M. R., Jablonowski, L., ... Holtfreter, B. (2015). Longitudinal effects of systemic inflammation markers on periodontitis. J Clin Periodontol, 42(11), 988-997. doi:10.1111/jcpe.12473

Romandini, M., Gioco, G., Perfetti, G., Deli, G., Staderini, E., \& Laforì, A. (2017). The association between periodontitis and sleep duration. Journal of clinical periodontology, 44(5), 490501.

Saletu, A., Pirker-Frühauf, H., Saletu, F., Linzmayer, L., Anderer, P., \& Matejka, M. (2005). Controlled clinical and psychometric studies on the relation between periodontitis and depressive mood. Journal of clinical periodontology, 32(12), 1219-1225.

Singh, V. P., Gan, J. Y., Liew, W. L., Soe, H. H. K., Nettem, S., \& Nettemu, S. K. (2019). Association between quality of sleep and chronic periodontitis: A case-control study in Malaysian population. Dental research journal, 16(1), 29.

Singla, N., Acharya, S., Prabhakar, R. V., Chakravarthy, K., Singhal, D., \& Singla, R. (2016). The impact of lifestyles on the periodontal health of adults in Udupi district: A cross sectional study. Journal of Indian Society of Periodontology, 20(3), 330.

Slots, J. (2017). Periodontitis: facts, fallacies and the future. Periodontol 2000, 75(1), 7-23. doi:10.1111/prd.12221 
Smagula, S. F., Stone, K. L., Fabio, A., \& Cauley, J. A. (2016). Risk factors for sleep disturbances in older adults: Evidence from prospective studies. Sleep Med Rev, 25, 21-30. doi:10.1016/j.smrv.2015.01.003

Suh, J. S., Kim, S., Bostrom, K. I., Wang, C. Y., Kim, R. H., \& Park, N. H. (2019). Periodontitisinduced systemic inflammation exacerbates atherosclerosis partly via endothelialmesenchymal transition in mice. Int J Oral Sci, 11(3), 21. doi:10.1038/s41368-019-00541

Vgontzas, A. N., Fernandez-Mendoza, J., Liao, D., \& Bixler, E. O. (2013). Insomnia with objective short sleep duration: the most biologically severe phenotype of the disorder. Sleep medicine reviews, 17(4), 241-254.

Wiener, R. C. (2016). Relationship of routine inadequate sleep duration and periodontitis in a nationally representative sample. Sleep disorders, 2016.

Yaggi, H. K., Araujo, A. B., \& McKinlay, J. B. (2006). Sleep duration as a risk factor for the development of type 2 diabetes. Diabetes Care, 29(3), 657-661.

Yoshida, Y., Ogawa, Y., Imaki, M., Nakamura, T., \& Tanada, S. (1997). Lifestyles and periodontal disease of Japanese factory workers. Environmental health and preventive medicine, 1(4), 188-192. 